\title{
Magnesium Status in Children with Attention-Deficit/Hyperactivity Disorder and/or Autism Spectrum Disorder
}

\author{
Anatoly V. Skalny ${ }^{1,2,3}$, Anna L. Mazaletskaya ${ }^{1}$, Olga P. Ajsuvakova ${ }^{1,2,3}$, Geir Bjørklund ${ }^{4}$, \\ Margarita G. Skalnaya ${ }^{2,3}$, Lyubov N. Chernova ${ }^{3}$, Andrey A. Skalny ${ }^{1,3}$, and Alexey A. Tinkov ${ }^{1,2,3}$ \\ ${ }^{1}$ Laboratory of Biotechnology and Applied Bioelementology, Yaroslavl State University, Yaroslavl, Russia \\ ${ }^{2}$ Laboratory of Molecular Dietetics, IM Sechenov First Moscow State Medical University, Moscow, Russia \\ ${ }^{3}$ Department of Medical Elementology, RUDN University, Moscow, Russia \\ ${ }^{4}$ Council for Nutritional and Environmental Medicine, Mo i Rana, Norway
}

Objective: The objective of this study was to assess serum, hair, and urinary magnesium (Mg) levels in children with attention-deficit/ hyperactivity disorder (ADHD), autism spectrum disorder (ASD), and both ASD and ADHD to reveal potential interactive effects.

Methods: A total of 148 boys aged 4-9 years old were enrolled in this study, including 44 children with ADHD, 40 pediatric patients with ASD, 32 patients with both ADHD and ASD, as well as 32 healthy neurotypical children. Hair, serum, and urinary Mg levels were assessed using inductively-coupled plasma mass spectrometry (ICP-MS). Laboratory quality control was performed using certified reference materials of human hair, plasma, and urine.

Results: No significant group difference in serum $\mathrm{Mg}$ levels was observed. $\mathrm{Mg}$ content in hair was found to be reduced in children with ADHD and ADHD+ASD compared to that in healthy controls by $11 \%$ and 15\%, respectively. Urinary Mg levels in children with ADHD+ASD exceeded the control, ADHD, and ASD values by 51, 76, and 65\%, respectively. Factorial analysis revealed significant contribution of $\mathrm{ADHD}$ to hair and urinary $\mathrm{Mg}$ levels. Multiple regression analysis demonstrated that hair and urinary Mg levels were considered as significant predictors of neurodevelopmental disorder complexity.

Conclusion: We propose that impaired Mg status may provide a link between ADHD and ASD.

Key Words: Neurodevelopmental disorders; Magnesium; Comorbidity; Urine; Hair.

Received: October 28, 2019 / Revision: December 4, 2019 / Accepted: December 16, 2019

Address for correspondence: Alexey A. Tinkov, Laboratory of Biotechnology and Applied Bioelementology, Yaroslavl State University, 14 Sovetskaya, Yaroslavl 150003, Russia

Tel: +7-961-9378198, Fax: +7-4852-797751, E-mail: tinkov.a.a@gmail.com

\section{INTRODUCTION}

Neurodevelopmental disorders, including attention-deficit/ hyperactivity disorder (ADHD) and autism spectrum disorder (ASD), represent a broad spectrum of pathologies characterized by disruption of brain development [1]. With a prevalence of up to 5\% [2] and 2\% [3], respectively, ADHD and ASD appear to be the most prevalent neurodevelopmental disorders with a higher incidence in males [4]. Despite a distinct clinical appearance, ADHD and ASD are characterized by certain similarities including impaired sensory processing, sleep disorders, and anxiety [5]. Moreover, ADHD frequently co-occurs in ASD [6]. It is proposed that ADHD and ASD may represent a single pathogenetic continuum [5].

This is an Open Access article distributed under the terms of the Creative Commons Attribution Non-Commercial License (https://creativecommons.org/licenses/by-nc/4.0) which permits unrestricted non-commercial use, distribution, and reproduction in any medium, provided the original work is properly cited.
Environmental factors [7], including nutrition [8], play a significant role in neurodevelopmental disorders. Particularly, children with ADHD [9] and ASD [10] are characterized by an impaired nutritional status. Existing data demonstrate a potential role of trace element, mineral, vitamin, and other micronutrient deficiencies in neurodevelopmental disorders due to their function in brain development [11].

Magnesium (Mg) is an essential element for child growth, and the recommended daily allowances for children aged 1-3, $4-8$, and 9-13 years old are estimated at 80,130 , and $240 \mathrm{mg}$, respectively. Mg regulation occurs through a balance between absorption, excretion, and redistribution from bones, and the element is involved in multiple processes [12] including brain development and functioning [13]. Multiple studies have assessed Mg status in ADHD, although with conflicting results at times [14,15]. However, data on Mg levels in children with ASD are limited. Mg content in the hair has been shown to 
be inversely associated with autism severity in an Indian cohort of children with ASD [16]. It is proposed that impaired $\mathrm{Mg}$ status may be associated with both ADHD and ASD [17]. Moreover, Mg supplements are also used as a complementary therapy in children with both ADHD [18] and ASD [19]. However, the potential interactive effects of ADHD and ASD on $\mathrm{Mg}$ status have not been studied.

In view of ADHD and ASD comorbidity, as well as the role of $\mathrm{Mg}$ in both ADHD and ASD, it is hypothesized that impaired Mg status may provide a link between these neurodevelopmental disorders, explaining the occurrence of hyperactivity in ASD. Therefore, the objective of the study was to assess serum, hair, and urinary Mg levels in children with ADHD, ASD, and both ADHD and ASD.

\section{METHODS}

The protocol of the study was approved by the Local Institutional Ethics Committee at Yaroslavl State University (Yaroslavl, Russia; approval no. 12/3-21.12.2018) in agreement with the principles of the Declaration of Helsinki (1964-2013). All children and their parents participated in the investigation on a voluntary basis. The informed consent form was signed by the parents. All procedures including anthropometric hair and blood sampling were performed in the presence of one of the parents.

A total of 148 boys aged 4-9 years old were enrolled in the present study including 44 children with ADHD, 40 pediatric patients with ASD, 32 patients with both ADHD and ASD, as well as 32 healthy neurotypical children. No significant group difference in age, weight, height, and body mass index (BMI) was observed between the study groups (Table 1). The data on ADHD (ICD-10: F90.0) and ASD (ICD-10: F84.0) diagnosis were extracted from the children's clinical records. ADHD was diagnosed using ICD-10 recommendations including inattention, hyperactivity, and impulsivity (not less than 3 symptoms of each). The Childhood Autism Rating Scale (CARS) was also used for validation of ASD diagnosis. ASD was also diagnosed using the following ICD-10 criteria: impaired development before the age of 3 years; impaired social interaction; abnormalities in communication; restricted, repetitive, and stereotyped behavior; and interests not attributable to other pervasive developmental disorders.

Blood serum was obtained through blood centrifugation at 1,600×g for 10 minutes with an EBA 200 (Hettich, Kirchlengern, Germany) centrifuge. Urine samples were collected by the parents using Vacuette ${ }^{\circledR}$ urine collection cups (Greiner Bio-One International AG, Kremsmünster, Austria). The proximal parts of hair strands $(0.05-0.10 \mathrm{~g})$ were collected using ethanol-precleaned stainless steel scissors from the occipital region with subsequent acetone-water washing.

Serum and urine samples were diluted with a diluent (1:15 v/v) containing (v/v) 1\% 1-butanol (Merck KGaA, Darmstadt, Germany) and 0.1\% Triton X-100 (Sigma-Aldrich, Co., St. Louis, MO, USA) in 18.2 M $\Omega$ distilled deionized water (Labconco Corp., Kansas City, MO, USA) acidified with 0.07\% HNO3 (Sigma-Aldrich, Co.) $(\mathrm{pH}=2)$. The hair samples were subjected to microwave digestion in Berghof SpeedWave- 4 (Berghof Products \& Instruments, Eningen, Germany).

Hair, serum, and urinary Mg levels were assessed using inductively-coupled plasma mass spectrometry (ICP-MS) at NexION 300D (PerkinElmer Inc., Waltham, MA, USA) equipped with ESI SC-2 DX4 autosampler (Elemental Scientific Inc., Omaha, NE, USA). The certified reference materials of human hair GBW09101 (SINR, Shanghai, China), plasma (ClinChek ${ }^{\circledR}$ Plasma Control, RECIPE Chemicals+ Instruments $\mathrm{GmbH}$, Munich, Germany) and urine (ClinChek ${ }^{\circledR}$ Urine Control) were used for laboratory quality control.

Statistical analysis was performed using Statistica 10.0 software (StatSoft, Tulsa, OK, USA). Distribution normality was assessed using Shapiro-Wilk and Kolmogorov-Smirnov tests. Data on hair, serum, and urine Mg levels were expressed as median and the respective interquartile range (IQR) due to skewed distribution. The initial values were log-transformed for further processing with repeated distribution analysis. Group comparisons were performed using analysis of covariance (ANCOVA) adjusted for age and anthropometric parameters (weight, height, BMI) with subsequent Bonferroni correction for multiple testing for the group mean comparisons of log-transformed serum and hair Mg levels. As the log-

Table 1. Age and anthropometric parameters of the examined children

\begin{tabular}{|c|c|c|c|c|c|c|c|}
\hline \multirow{2}{*}{ Parameter } & \multirow{2}{*}{ Control } & \multirow{2}{*}{ ADHD } & \multirow{2}{*}{ ASD } & \multirow{2}{*}{$A D H D+A S D$} & \multicolumn{3}{|c|}{$\mathrm{p}$ values ANOVA } \\
\hline & & & & & ADHD & ASD & ADHD $\times A S D$ \\
\hline Age $(y r)$ & $5.69 \pm 1.38$ & $5.86 \pm 1.72$ & $5.48 \pm 1.45$ & $5.38 \pm 1.31$ & 0.201 & 0.289 & 0.213 \\
\hline Weight $(\mathrm{kg})$ & $22.58 \pm 4.90$ & $23.07 \pm 6.25$ & $25.41 \pm 14.20$ & $22.59 \pm 7.95$ & 0.855 & 0.905 & 0.413 \\
\hline Height $(\mathrm{cm})$ & $118.90 \pm 11.60$ & $119.70 \pm 10.91$ & $119.74 \pm 17.29$ & $118.09 \pm 14.09$ & 0.521 & 0.380 & 0.802 \\
\hline $\mathrm{BMI}\left(\mathrm{kg} / \mathrm{m}^{2}\right)$ & $15.95 \pm 2.60$ & $15.84 \pm 2.15$ & $16.71 \pm 3.50$ & $15.89 \pm 2.91$ & 0.503 & 0.486 & 0.477 \\
\hline
\end{tabular}

Data are expressed as mean \pm standard deviation; no significant group difference was observed $(p>0.05)$. ADHD: attention-deficit/hyperactivity disorder, ASD: autism spectrum disorder, BMI: body mass index, ANOVA: analysis of variance 
transformed data on urinary Mg levels did not reach log-normal distribution, group comparison was performed using nonparametric Kruskal-Wallis test with adjustment for multiple comparisons. Factorial analysis was performed using two-way ANCOVA using log-transformed data. The results of the tests were considered significant at $\mathrm{p}<0.05$.

\section{RESULTS}

The obtained data showed that the patterns of Mg status depended significantly on the studied samples (Fig. 1). No significant group difference in serum $\mathrm{Mg}$ levels was observed when compared to that in the control group. Hair Mg content was found to be reduced in children with ADHD and ADHD+ASD compared to the controls by $11 \%$ and $15 \%$, respectively. It is notable that no significant group difference in hair $\mathrm{Mg}$ content was observed between children with ADHD or ASD. Urinary Mg levels in children with ADHD+ASD exceeded the control, ADHD, and ASD values by 51,76 , and $65 \%$, respectively.

Factorial analysis using two-way ANCOVA (Table 2) demonstrated that although ADHD and ASD did not affect serum $\mathrm{Mg}$ levels, factorial interaction (ADHD $\times$ ASD) had a nearly significant effect $(\mathrm{p}<0.10)$. In contrast, ADHD had a significant effect on hair and urinary Mg levels, whereas ASD did not have any factorial influence.

Correlation analysis demonstrated that only hair Mg content was weakly but significantly associated with age values $(r=0.163 ; p=0.026)$, whereas no other significant associations with anthropometric parameters were revealed (data not shown).

\section{DISCUSSION}

This study provides unique data on comparative analysis of $\mathrm{Mg}$ status in children with ADHD and/or ASD. Generally, the revealed associations between hair (inverse) and urinary (direct) Mg levels and neurodevelopmental disorders may be indicative of increased $\mathrm{Mg}$ excretion in children with ADHD and ASD, ultimately leading to a reduced $\mathrm{Mg}$ burden in the body. The lack of significant alterations in serum $\mathrm{Mg}$ levels may occur due to homeostatic regulation aimed at maintenance of circulating $\mathrm{Mg}$ levels through modulation of absorbance, excretion, and tissue redistribution (especially bones) [20].

The current findings generally agree with the earlier indications of altered Mg status in ADHD and ASD. Particularly, $\mathrm{Mg}$ deficiency was found to be prevalent in 65\% of examined Egyptian children with ADHD and also correlated with impulsivity, hyperactivity, and inattention [21]. Moreover, the first detailed study of $\mathrm{Mg}$ status in ADHD also revealed $\mathrm{Mg}$ deficiency in 95\% of children with ADHD [22]. Results of the most recent meta-analysis have demonstrated that children with ADHD have $0.105 \mathrm{mmol} / \mathrm{L}$ lower serum $\mathrm{Mg}$ levels than in neurotypical controls [15]. Furthermore, hair Mg content
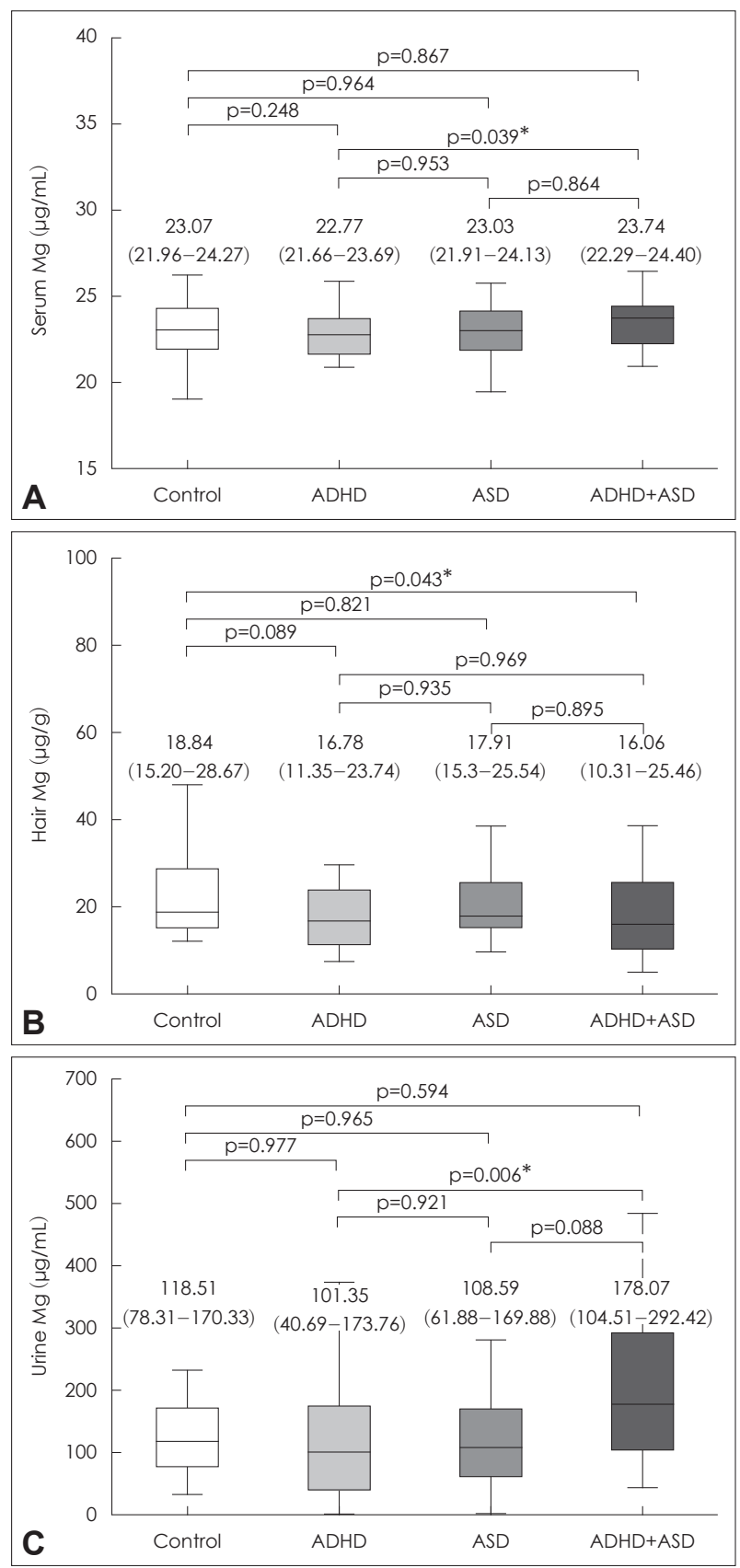

Fig. 1. Serum (A), hair (B), and urinary (C) magnesium levels in children with $A D H D, A S D$, as well as neurotypical controls. Data expressed as median (line), IQR (box), and non-outlier range (whiskers). $\mathrm{P}$ values are provided according to Bonferroni corrected ANCOVA (A, B) or Kruskal-Wallis test (C), being considered significant at $* p<0.05$. ADHD: attention-deficit/hyperactivity disorder, ASD: autism spectrum disorder, IQR: interquartile range, ANCOVA: analysis of covariance, Mg: magnesium. 
Table 2. Assessment of the impact of ADHD, ASD, or factorial interaction (ADHD $\times$ ASD) on serum, hair, and urinary Mg levels

\begin{tabular}{lccc}
\hline \multicolumn{1}{c}{ Factor } & Serum Mg & Hair Mg & Urine Mg \\
\hline ASD & 0.482 & 0.604 & 0.151 \\
ADHD & 0.395 & $0.025^{*}$ & $0.021^{*}$ \\
ADHD $\times$ ASD & 0.057 & 0.913 & 0.532 \\
\hline
\end{tabular}

Data expressed as $\mathrm{p}$ values; ${ }^{*}$ the factorial effect is significant at $p<0.05$. ADHD: attention-deficit/hyperactivity disorder, ASD: autism spectrum disorder, Mg: magnesium

is also lower in children with ADHD than in healthy children, as estimated by another recent meta-analysis [23]. In contrast to the above, Irmisch et al. [14] demonstrated that the blood serum Mg levels in German children with ADHD exceeded the control values. Although certain indications of the potential effects of Mg supplementation in ADHD exist, recent meta-analyses demonstrate the lack of systematic data [19].

Indications of altered $\mathrm{Mg}$ status in ASD are less prevalent. Particularly, a significant severity-associated decrease in hair Mg levels was observed in Indian children with ASD [16]. A recent meta-analysis demonstrated reduced hair Mg levels in ASD [24]. Examination of a limited sample of Italian children with ASD demonstrated that plasma Mg levels were significantly lower in children with autism than in healthy controls [25]. Correspondingly, examination of Nigerian children with ASD revealed a significant $25 \%$ decrease in serum $\mathrm{Mg}$ compared to neurotypical controls [26]. We have also revealed that serum Mg levels in ASD are inversely associated with neuroinflammation [27]. The observed lack of increase in serum or hair Mg levels in children with ASD, at least partially, corresponds to the observation of higher $\mathrm{Mg}$ intake in autistic children [28]. Although certain indications of $\mathrm{Mg}$ supplementation efficiency in ASD exist, no systematic data are available [18].

$\mathrm{Mg}$ is considered as an important player in neurodevelopment and neuronal maturation [13] and may modulate the pathogenetic mechanisms of both ADHD and ASD development. Particularly, Mg treatment can reduce neuroinflammation [27] and excitotoxicity [28]. It may also interact with dopamine signaling, thus providing a link between Mg status and ADHD [29]. Moreover, adequate Mg levels support plasticity and interconnectivity [13].

This study had a few limitations worth mentioning. First, the disease severity was not monitored, although it could be beneficial to reveal any association between $\mathrm{Mg}$ status and adverse neurodevelopment. Moreover, monitoring of $\mathrm{Mg}$ dietary intake could have provided additional insight into the causes of the observed alterations.

\section{CONCLUSION}

The obtained data demonstrate that children with both ASD and ADHD are characterized by more pronounced alterations in hair and urinary $\mathrm{Mg}$ levels compared to healthy control children. An impaired Mg status may predispose children to more complex neurodevelopmental disorders including ADHD and ASD comorbidity. However, further studies are required to clarify the mechanisms linking impaired $\mathrm{Mg}$ metabolism and neurodevelopmental disorders.

\section{Acknowledgments}

The project was supported by the Russian Foundation for Basic Research (RFBR) No. 19-013-00528.

\section{Conflicts of Interest}

The authors have no potential conflicts of interest to disclose.

\section{Author Contributions}

Conceptualization: Anatoly V. Skalny, Alexey A. Tinkov. Data curation: Anna L. Mazaletskaya, Geir Bjørklund. Formal analysis: Anna L. Mazaletskaya, Geir Bjørklund. Funding acquisition: Alexey A. Tinkov. Investigation: Andrey A. Skalny, Olga P. Ajsuvakova, Margarita G. Skalnaya, Lyubov N. Chernova. Methodology: Andrey A. Skalny, Olga P. Ajsuvakova, Margarita G. Skalnaya, Lyubov N. Chernova. Project administration: Alexey A. Tinkov. Resources: Anatoly V. Skalny, Margarita G. Skalnaya. Software: Anna L. Mazaletskaya, Geir Bjørklund. Supervision: Anatoly V. Skalny, Alexey A. Tinkov. Validation: Anatoly V. Skalny, Margarita G. Skalnaya, Alexey A. Tinkov. Visualization: Alexey A. Tinkov. Writingoriginal draft: Anna L. Mazaletskaya, Andrey A. Skalny, Olga P. Ajsuvakova, Lyubov N. Chernova. Writing-review \& editing: Anatoly V. Skalny, Margarita G. Skalnaya, Alexey A. Tinkov.

\section{ORCID iDs}

Anna L. Mazaletskaya https://orcid.org/0000-0002-1108-5949

Olga P. Ajsuvakova https://orcid.org/0000-0003-4707-9353

Geir Bjørklund https://orcid.org/0000-0003-2632-3935

Margarita G. Skalnaya https://orcid.org/0000-0003-1099-2560

Lyubov N. Chernova https://orcid.org/0000-0002-5498-1807

Andrey A. Skalny https://orcid.org/0000-0001-5310-3853

Alexey A. Tinkov https://orcid.org/0000-0003-0348-6192

\section{REFERENCES}

1) Thapar A, Cooper M, Rutter M. Neurodevelopmental disorders. Lancet Psychiatry 2017;4:339-346.

2) Sayal K, Prasad V, Daley D, Ford T, Coghill D. ADHD in children and young people: prevalence, care pathways, and service provision. Lancet Psychiatry 2018;5:175-186.

3) Fombonne E. The rising prevalence of autism. J Child Psychol Psychiatry 2018;59:717-720.

4) Doernberg E, Hollander E. Neurodevelopmental disorders (ASD and ADHD): DSM-5, ICD-10, and ICD-11. CNS Spectr 2016;21: 295-299.

5) Kern JK, Geier DA, Sykes LK, Geier MR, Deth RC. Are ASD and ADHD a continuum? A comparison of pathophysiological similarities between the disorders. J Atten Disord 2015;19:805-827.

6) Leitner Y. The co-occurrence of autism and attention deficit hyperactivity disorder in children - what do we know? Front Hum Neurosci 2014;8:268. 
7) Pietropaolo S, Crusio WE, Feldon J. Gene-environment interactions in neurodevelopmental disorders. Neural Plast 2017;2017: 9272804.

8) Bhutta ZA, Guerrant RL, Nelson CA 3rd. Neurodevelopment, nutrition, and inflammation: the evolving global child health landscape. Pediatrics 2017;139(Suppl 1):S12-S22.

9) Greenblatt JM, Delane DD. Micronutrient deficiencies in ADHD: a global research consensus. J Orthomol Med [serial online] 2017; 32(6). Available from URL: https://isom.ca/article/micronutrientdeficiencies-adhd-global-research-consensus/.

10) Hyman SL, Stewart PA, Schmidt B, Cain U, Lemcke N, Foley JT, et al. Nutrient intake from food in children with autism. Pediatrics 2012;130 Suppl 2:S145-S153.

11) González HF, Visentin S. Micronutrients and neurodevelopment: an update. Arch Argent Pediatr 2016;114:570-575.

12) Cao S, Hodges JK, McCabe LD, Weaver CM. Magnesium requirements in children recommendations for reevaluation and comparison with current evidence for adults. Nutr Today 2019;54:195-206.

13) Yamanaka R, Shindo Y, Oka K. Magnesium is a key player in neuronal maturation and neuropathology. Int J Mol Sci 2019;20: E3439.

14) Irmisch G, Thome J, Reis $\mathbf{O}$, Hässler F, Weirich S. Modified magnesium and lipoproteins in children with attention deficit hyperactivity disorder (ADHD). World J Biol Psychiatry 2011;12 Suppl 1:63-65.

15) Effatpanah M, Rezaei M, Effatpanah H, Effatpanah Z, Varkaneh HK, Mousavi SM, et al. Magnesium status and attention deficit hyperactivity disorder (ADHD): a meta-analysis. Psychiatry Res 2019;274:228-234.

16) Priya MDL, Geetha A. Level of trace elements (copper, zinc, magnesium and selenium) and toxic elements (lead and mercury) in the hair and nail of children with autism. Biol Trace Elem Res 2011; 142:148-158.

17) Mousain-Bosc M, Siatka C, Bali JP. Magnesium, hyperactivity and autism in children. In: Vink R, Nechifor M, editors. Magnesium in the central nervous system [Internet]. Adelaide: University of Adelaide Press;2011
18) Hariri M, Azadbakht L. Magnesium, iron, and zinc supplementation for the treatment of attention deficit hyperactivity disorder: a systematic review on the recent literature. Int J Prev Med 2015;6:83.

19) Murza KA, Pavelko SL, Malani MD, Nye C. Vitamin B6-magnesium treatment for autism: the current status of the research. Magnes Res 2010;23:115-117.

20) Elbaz F, Zahra S, Hanafy H. Magnesium, zinc and copper estimation in children with attention deficit hyperactivity disorder (ADHD). Egypt J Med Hum Genet 2017;18:153-163.

21) de Baaij JH, Hoenderop JG, Bindels RJ. Regulation of magnesium balance: lessons learned from human genetic disease. Clin Kidney J 2012;5(Suppl 1):i15-i24.

22) Kozielec T, Starobrat-Hermelin B. Assessment of magnesium levels in children with attention deficit hyperactivity disorder (ADHD). Magnes Res 1997;10:143-148.

23) Huang YH, Zeng BY, Li DJ, Cheng YS, Chen TY, Liang HY, et al. Significantly lower serum and hair magnesium levels in children with attention deficit hyperactivity disorder than controls: A systematic review and meta-analysis. Prog Neuropsychopharmacol Biol Psychiatry 2019;90:134-141.

24) Saghazadeh A, Ahangari N, Hendi K, Saleh F, Rezaei N. Status of essential elements in autism spectrum disorder: systematic review and meta-analysis. Rev Neurosci 2017;28:783-809.

25) Strambi M, Longini M, Hayek J, Berni S, Macucci F, Scalacci E, et al. Magnesium profile in autism. Biol Trace Elem Res 2006;109: 97-104.

26) Omotosho IO, Akinade AO, Lagunju IA. Calcium and magnesium levels are down regulated in Nigerian children with autism spectrum disorder and cerebral palsy. Neurosci Med 2018;9:159-170.

27) Skalny AV, Simashkova NV, Skalnaya AA, Klyushnik TP, Zhegalova IV, Grabeklis AR, et al. Trace element levels are associated with neuroinflammatory markers in children with autistic spectrum disorder. J Trace Elem Med Biol 2018;50:622-628.

28) Kirkland AE, Sarlo GL, Holton KF. The role of magnesium in neurological disorders. Nutrients 2018;10:E730.

29) Gröber U, Schmidt J, Kisters K. Magnesium in prevention and therapy. Nutrients 2015;7:8199-8226. 